\title{
Quaderni
}

QUADERNI Communication, technologies, pouvoir

97 | Automne 2018

Néo-libéralisme(s). Réseaux et formes des

mobilisations en France

\section{Le néolibéralisme dans les politiques culturelles : le discours des médiateurs du chèque culture en France}

\section{Arnaud Lacheret}

\section{OpenEdition}

\section{Journals}

Édition électronique

URL : http://journals.openedition.org/quaderni/1314

DOI : 10.4000/quaderni.1314

ISSN : 2105-2956

Éditeur

Les éditions de la Maison des sciences de l'Homme

Édition imprimée

Date de publication : 5 octobre 2018

Pagination : 89-106

\section{Référence électronique}

Arnaud Lacheret, «Le néolibéralisme dans les politiques culturelles : le discours des médiateurs du chèque culture en France », Quaderni [En ligne], 97 | Automne 2018, mis en ligne le 05 octobre 2020 consulté le 05 janvier 2021. URL : http://journals.openedition.org/quaderni/1314 ; DOI : https://

doi.org/10.4000/quaderni.1314 


\section{$D$ ossier}

\section{Le néolibéralisme dans les politiques culturelles :}

le discours des médiateurs du chèque culture en France

\section{Arnaud Lacheret}

Docteur en Science Politique Chercheur associé à PACTE, Université Grenoble Alpes

Sciences Po Grenoble. Directeur de la French Arabian Business School, Arabian Gulf University, Bahrain
Lors de la campagne présidentielle française de 2017, le candidat Emmanuel Macron promettait la création d'un «Pass culture » permettant aux « jeunes » d'accéder à «la culture ». Un an après l'élection, Françoise Nyssens, ministre de la culture, précisait les contours de cette aide de 500 euros qui serait créditée sur un compte virtuel lorsqu'un jeune atteindrait 18 ans ${ }^{1}$. Ce «Pass Culture » permettra à son bénéficiaire d'avoir accès à toute une gamme de biens et services culturels au sein d'un " marché » défini et limité par le gouvernement en lien avec les acteurs du monde de la culture.

Outre le côté « moderne », digital et « géolocalisé » promis par les concepteurs (un inspecteur des finances et un ancien d'HEC propriétaire notamment de Beaux Arts Magazine) travaillant « en mode start up » mandatés pour réaliser ce pass, on ne peut s'empêcher de faire l'analogie avec un instrument néolibéral fonctionnant déjà en France et à l'étranger lorsque l'on l'examine attentivement.

Cet instrument, qui présente de troublantes analogies avec le pass culture, est français, ce qui est d'autant plus surprenant que le gouvernement ne l'a jamais mentionné alors qu'il aurait été simple politiquement de s'en réclamer puisqu'il lui aurait permis de valoriser une initiative des collectivités locales. Il s'agit du chèque culture, instrument de politique culturelle créé en 1993-1994 au Conseil Régional Rhône-Alpes et qui s'est ensuite répandu, sous différentes formes, dans la plupart des régions françaises. Plusieurs études et évaluations ont en effet été menées à propos de ces chèques (Lacerenza, 2001, Saez et Lacheret, 2001, Lacheret, 2014), y compris par le ministère 
de la Culture (Rouet, 2009) inspirés eux-mêmes d'expérimentations ayant notamment eu lieu au tÉtats-Unis dans les années 1960 (Peacock, 1968, 1969) sans pour autant que les créateurs du pass culture ne s'appuient sur les résultats de ces travaux étudiant ces aides individuelles qui existent toujours pour la plupart.

Les chèques culture présents au sein des régions et départements français furent conçus, dans le même esprit que le pass culture, pour permettre à un public «ciblé» (les lycéens ou les collégiens) de bénéficier de possibilités d'accès à la pratique culturelle via une subvention individuelle fléchée (le chèque, qui peut être dématérialisé sous forme de carte ou d'application mobile). Concrètement, il s'agissait de leur fournir des bons d'achats sur des livres, des places pour assister à un spectacle vivant, des entrées au cinéma, des adhésions à des activités culturelles, des entrées dans des musées...

Lors de son lancement en 1993-1994, au conseil régional Rhône-Alpes, par la majorité dirigée par Charles Millon (membre du Parti Républicain, formant l'aile droite de l'UDF à l'époque), certains des promoteurs du chèque culture établissaient également une filiation avec les «vouchers » utilisés dans le monde anglo-saxon, particulièrement avec le "school voucher", conçu par Milton Friedman, censé favoriser le libre choix de l'école dans un système où l'éducation serait considérée comme un marché. Les entretiens menés à l'époque dans le cadre d'un mémoire de DEA témoignaient sans surprise de cette filiation qui incarnait, pour les acteurs, une " politique libérale de la culture $»^{2}$. Charles Millon lui-même revendiquait très naturellement cette filiation avec le «school voucher " anglosaxon $^{3}$.

L'objet de cet article est d'abord de questionner le discours des médiateurs politiques français du chèque qui a pu conduire à l'introduction d'un outil dont nous aurons démontré le caractère néolibéral, puis à lui faire rencontrer un immense succès puisqu'il fut massivement diffusé à travers les collectivités locales pour réapparaître à une échelle gouvernementale 25 ans après son lancement initial.

Nous nous attacherons donc dans un premier temps à définir en quoi le chèque est néolibéral, avant de rentrer dans l'analyse du discours des médiateurs ayant assumé sa dimension néolibérale. Enfin nous comparerons les résultats des études menées avec les non libéraux ayant assuré la diffusion du chèque après le lancement $\mathrm{du}$ pionnier rhônalpin.

Pour cela, nous définirons d'abord la notion de «voucher » et ses origines avant de mettre en évidence le sens qu'il porte et ses caractéristiques néolibérales. Nous interrogerons ensuite la construction du discours des acteurs politiques libéraux ayant introduit le chèque et tenterons de donner une explication à la transformation de ce discours au fil de la diffusion du chèque par leurs successeurs, des acteurs de collectivités gérées par la gauche ayant parfaitement adopté l'objet. Le processus de dépolitisation et de neutralisation du discours autour de cet instrument libéral est, en définitive, ce qui a permis au candidat Emmanuel Macron, puis au ministère de la Culture de pouvoir en faire un argument électoral, puis un instrument de politique publique, sans que son 
caractère néolibéral ne soit même évoqué, par la majorité comme pas l'opposition.

\section{Le « voucher », instrument néolibéral}

Le « voucher » (Colin, 2005), ou chèque, est souvent présenté comme un instrument d'action publique néolibéral eu égard à son origine et à ses caractéristiques propres. Conceptualisé par Milton Friedman (Friedman, 1962), bien que le bon d'achat ciblé soit un outil beaucoup plus ancien que l'on retrouve notamment sous l'Angleterre victorienne (Navailles, 1983), il tire de cette paternité une image néolibérale revendiquée qui cause encore de vifs débats politiques aux ÉtatsUnis. Selon Friedman, l'instruction obligatoire et publique est nécessaire car elle participe au bien-être de chacun des membres de la société. En revanche, il n'existerait aucun argument valide pour justifier le fait que le service soit nationalisé et assuré directement par l'État. Il est donc selon Friedman préférable de faire entrer une logique de marché dans le processus de sélection par les familles de l'école et du type de scolarité pour les enfants. Pour permettre de concilier l'objectif de l'instruction obligatoire, et accessible pour tous, et la liberté de choix, il a imaginé un "chèque éducation »d'un montant fixé par année et par famille qui permettrait à chacune d'entre elles de choisir parmi des écoles agréées par le gouvernement (mais qui peuvent être autonomes). Le « voucher » est notamment toujours l'un des principaux arguments politiques utilisé contre la secrétaire à l'Éducation américaine, Betsy $\mathrm{DeVos}^{4}$, et a fait l'objet d'évaluations fréquentes et engagées outre-Atlantique depuis plus de 20 ans (Lacheret, 2014, Mac Ewan, 2000) prouvant tantôt que cette méthode de paiement des aides individuelles était efficace (Greene, 1998, 2001, 2011), tantôt qu'elle était calamiteuse (Carnoy, 2008).

Si la paternité libérale du « voucher » anglo-saxon ne fait aucun doute et ne manque pas d'être soulignée dans toutes les études, le contexte français amène à davantage de prudence. À première vue, il apparaît dans les années 1990, à une période où la droite française vient d'accomplir son aggiornamento idéologique lors de son passage dans l'opposition, où les clubs, think tanks et associations parfois connectés à des réseaux internationaux (Brookes, 2012), ont permis à une partie du personnel politique français de droite de trouver une source d'inspiration à travers une démarche de benchmarking des expériences réalisées à l'étranger (Perrier, 2015). C'est l'époque où Alain Madelin, s'inspirant notamment de Friedman, vient d'écrire un ouvrage évoquant justement la possibilité de libéraliser le système scolaire (Madelin, 1984).

En France, le chèque a emprunté la voie des territoires avec notamment la multiplication des chèques culture dans les régions dont il fut démontré qu'il s'agissait d'un transfert dépolitisé d'instruments de politiques publiques (Lacheret, 2017). Il faut, pour mieux comprendre ce terme, remonter aux «policy transfer studies » (Marsh, Dolowitz, 2000) et l'appliquer aux instruments d'action publique (Lascoumes, Le Gales, 2004) dont le « voucher » est un exemple idéal (Peters, 2002, 2014).

La notion de «policy transfer » étudie le processus par lequel une politique publique passe d'un système à un autre en mobilisant notamment la 
notion de convergence politique (la tendance pour des sociétés à évoluer de la même façon, à développer des structures, processus et performances similaires).

Ce concept, qui étudiait de façon très large les États et groupes d'États a été rénové par David Marsh et David Dolowitz qui y voient le processus par lequel les informations sur une politique publique, les procédures administratives, institutions et idées d'un système politique (passé ou présent) sont utilisées pour développer des politiques publiques, des procédures administratives, des institutions ou des idées dans un autre système politique. Appliquée à un instrument d'action publique 5 , la notion de policy transfer permet de comprendre à la fois l'importation de l'instrument en France mais également sa diffusion au sein des collectivités locales, puis sa reprise par le gouvernement.

La démonstration de la dépolitisation de cet instrument et de sa technicisation a été faite dans le contexte français (Lacheret, 2014) et a repris les conclusions de plusieurs auteurs américains sur le sujet (Salamon, 2002, Steuerle, 2000) qui ne voyaient dans le chèque qu'une disposition technique, un outil remplaçable par un autre outil sans réel sens politique. Outre-Atlantique, cette volonté de la part des chercheurs anglosaxons de dépolitiser le chèque apparaît comme surprenante, comme si la parenté friedmanienne du chèque n'était pas assumée. Il est vrai, en observant les travaux effectués dans les années 1990 que «l'inventeur » du « voucher $»$ a tendance à être éclipsé au point de n'apparaitre qu'à cinq reprises dans le corps du texte dans les 552 pages de l'ouvrage dirigé par Eugene Steuerle en 2000,
Vouchers and the provision of public service, ce qui est déjà beaucoup puisque deux ans plus tard, le nom de Friedman n'apparaît aucune fois, pas même en bibliographie, dans l'ouvrage de Lester Salamon, The Tools of Government (Salamon, 2002).

Or ces recherches ne rendent pas bien compte du fait que les premiers importateurs politiques du chèque en France en assumaient la paternité néolibérale peu de temps après que les droites se fussent intéressées aux modèles reaganiens et thatchériens. Un parallèle intéressant peut d'ailleurs être fait entre la redéfinition de cet instrument aux États-Unis, puis sa réappropriation, notamment par les Républicains qui voit disparaître toute référence au néolibéralisme et sa généralisation en France, accompagnée d'une neutralisation et donc d'une disparition de son caractère néolibéral après qu'il ait été abondamment revendiqué lors de la première introduction du chèque. Aux États-Unis toutefois, cette redéfinition du chèque, s'est accompagnée de soubresauts militants assez importants dénonçant ce caractère néolibéral et cette réappropriation silencieuse a été largement commentée (Weil, 2002, Baltodano, 2012).

En France, cette disparition des origines néolibérales du chèque est assez étrange puisque les entretiens menés ont clairement indiqué que les acteurs contemporains ${ }^{6}$ du chèque (interrogés à partir de 2010) y accordaient un rôle «utilitariste » dépourvu de sens politique (Lacheret, 2016). Toutefois, et c'est le principal élément de problématique de cet article, des entretiens menés de 1999 à 2013 auprès des élus et fonctionnaires pionniers du chèque (ceux qui ont implanté 
la première version en 1993-1994) en France montrent qu'ils assumaient, et assument toujours, une filiation nettement néolibérale. L'intérêt de cet article est donc de croiser les entretiens des pionniers de cet instrument, avec ceux qui l'utilisent 25 ans après et ainsi de comprendre comment le discours a évolué tandis que l'instrument est resté.

Au total, 45 entretiens semi-directifs ont été menés auprès de 16 élus ( 7 en 2000-2001 et 9 entre 2010 et 2013), 23 fonctionnaires territoriaux (dont 7 évoluant à des fonctions de direction générale) entre 2010 et 2013, et 6 acteurs de sociétés privées en charge de commercialiser les chèques (entre 2010 et 2013). L'objectif des entretiens était notamment d'obtenir une définition du chèque afin de la comparer avec les principaux traits caractéristiques relevés dans les travaux anglo-saxons sur le sujet.

Les entretiens avec les médiateurs se revendiquant néolibéraux portent sur une dizaine d'élus locaux ayant participé à la mise en place du chèque culture Rhône-Alpes en 1994, qui est considéré comme le premier dispositif de type «voucher » introduit en France de façon pérenne et qui fut ensuite abondamment diffusé parmi les collectivités pour distribuer diverses aides individuelles, allant bien au-delà de la simple politique culturelle (Colin, 2005). La première série d'entretiens fut réalisée entre 1999 et 2001. $\mathrm{Au}$ cours des entretiens réalisés notamment avec l'ancien président du Conseil régional Charles Millon, le vice-président en charge de la culture Hervé Mariton ou le président de la commission Culture Amaury Nardonne, il n'était pas initialement question d'évoquer le sujet de la filiation libérale, que cela soit lors des rencontres en 2000-2001 (mémoire de DEA) ou en 2010-2013 (thèse de doctorat) mais cette dernière s'est «invitée» dans les propos des acteurs. L'objectif des entretiens était de comprendre d'où venait l'idée du chèque et de tester plusieurs arguments pouvant justifier sa mise en place pour les comparer ensuite avec ceux des chercheurs et praticiens anglo-saxons.

Il a donc été nécessaire, pour bâtir cet article, de relire les transcriptions et de les analyser sous l'angle des arguments néolibéraux afin de comprendre quel discours était mobilisé par les élus revendiquant l'approche néolibérale.

Parmi la cinquantaine d'entretiens réalisés, certains acteurs, les médiateurs néolibéraux, ont adopté un discours qui ne correspondait pas aux hypothèses de départ puisque plutôt que de ne présenter le chèque que sous un angle fonctionnel, ils ont eu tendance à revendiquer sa filiation libérale tout en citant les différents arguments et marqueurs définis par les chercheurs anglosaxons (Steuerle, 2000, Salamon, 2002, Sturgess et Bodroza, 2011). Ils proviennent d'élus ou d'anciens élus rhônalpins de la droite libérale ayant eu des responsabilités dans le lancement et la promotion du chèque culture. Une première lecture des retranscriptions laisse apparaître une structure discursive originale, qui nous permettra d'émettre des hypothèses sur la façon dont les outils néolibéraux ont pu être introduits par des médiateurs en revendiquant le caractère néolibéral.

Nous tenterons donc de démontrer, grâce à une analyse par les instruments, que le néolibéralisme 
est arrivé, au moins partiellement, par le biais des territoires avec l'aide de médiateurs qui ont su développer un argumentaire et une structure discursive qui fut ensuite reprise et dépolitisée par d'autres acteurs politiquement éloignés du néolibéralisme.

\section{Quel néolibéralisme pour le chèque ?}

L'analyse du discours des acteurs permet de mieux comprendre de quelle famille du néolibéralisme ils se revendiquent sans réellement la mentionner. Tout d'abord, le terme « néolibéralisme » en tant que tel n'est prononcé qu'à une seule reprise par Charles Millon au cours des entretiens, celui d'ordolibéralisme est cité par Amaury Nardonne (la définition qu'il en donne, sur laquelle nous reviendrons, est pour le moins personnelle et originale). Les acteurs préfèrent parler de libéralisme, ou de politique libérale, mettant en avant la notion de liberté ou de libre choix. Cependant, nous ne parlons pas ici d'un instrument visant à libéraliser un secteur puisque dans l'esprit des élus interrogés, il n'est pas question de ne plus subventionner la culture mais simplement d'en changer la méthode de financement. Nous verrons que les acteurs considèrent tous qu'en matière de culture, ce n'est pas à l'autorité publique de faire des choix, mais qu'il faut donner la possibilité au public de choisir et donc de subventionner l'institution qu'il souhaite. On tend donc à introduire un mécanisme de marché dans une politique publique sans pour autant cesser de la soutenir au moyen d'argent public (on remet cette somme entre les mains du public afin qu'il choisisse lui-même qui subventionner, pour autant, la dépense publique reste identique).
La notion semble donc pour le moins parcellaire et pour la définir au mieux afin de la traduire en politiques publiques, nous avons choisi une approche plus instrumentale au sens des instruments d'action publique (Lascoumes, Le Gales, 2007) ou des «tools of public policy» (Salamon, 2002).

\section{Chèque éducation et quasi-marchés}

La notion de néolibéralisme rapportée à un instrument, a fortiori au chèque éducation, se nourrit de références plus anciennes que celles de Milton Friedman, qui réintroduit le concept dans Capitalism and Freedom en 1962. On peut remonter notamment à John Stuart Mill (1860), qui évoque déjà, pour un contexte britannique, l'importance du choix des parents pour l'éducation que pourraient recevoir leurs enfants. Hayek puis Friedman, sont par la suite les promoteurs américains de ce que Michel Foucault définit comme une technique de gouvernement de soi et des autres visant à étendre l'emprise des mécanismes du marché aux individus et à la société tout entière (Foucault, 1978).

Dans sa leçon sur la biopolitique, Foucault indique que dans le néolibéralisme, le marché est d'abord un milieu concurrentiel où l'individu n'est plus simplement une partie de l'échange, mais devient un véritable entrepreneur de luimême. Appliquée aux institutions éducatives et aux instruments de gestion, cette conception issue du corpus néolibéral permet d'introduire et d'étendre à d'autres sphères le concept de quasimarché (Glennester, Le Grand, 1995). Sont ainsi transférées les logiques du modèle marchand au monde de l'enseignement public dans une pers- 
pective de réforme en profondeur de l'éducation aux États-Unis. La démarche propose une forme de régulation alternative, marchande et individualisée, qui peut se suppléer aux financements étatiques des systèmes éducatifs publics considérés comme trop coûteux. L'octroi d'un chèque/ voucher peut ainsi se substituer, pour partie, aux financements éducatifs et servir de modèle de compromis entre des subventions massives publiques et un système totalement privé. C'est dans ce nouveau contexte dérégulé que l'usage du chèque s'est développé.

Dans une perspective néolibérale, il faut donc que la puissance publique n'intervienne que dans le but de permettre à l'individu-entrepreneur d'exercer son libre choix et de faire que le plan d'action personnel qu'il a retenu se concrétise. L'État doit donc garantir le bon fonctionnement des mécanismes de marché et permettre à l'individu de sélectionner les offres de formation de son choix.

\section{Une structure discursive ternaire chez les pionniers libéraux du chèque en France}

La méthodologie utilisée pour analyser les entretiens s'inscrit dans l'approche discursive. Cette approche considère le discours (qu'il faut ici comprendre comme la façon de mobiliser un argumentaire politique) comme la clé centrale de compréhension de la façon dont les acteurs construisent et infléchissent les politiques publiques. Ainsi, le discours est, tout d'abord, ce par quoi les acteurs conçoivent le monde. Il est aussi le moyen qu'ils utilisent pour le transformer. Il est enfin ce par quoi ils interagissent les uns avec les autres pour s'accorder tout autant que pour lutter. (Durnova, Zittoun, 2013).
L'analyse du discours des premiers acteurs français à avoir importé le chèque dans une politique locale et revendiquant explicitement leurs idées libérales permet de distinguer une structure assez identique menant au bout du compte à la justification et à la mise en place du chèque. Ainsi, la légitimation du chèque passe, chez ses promoteurs, par deux premières phases d'argumentation qui sont d'abord la nécessité de cibler un public bien déterminé, puis de lui offrir la possibilité de choisir librement. Ces deux premières phases débouchent ensuite sur la revendication libérale, formant un rythme ternaire systématique chez les pionniers libéraux du chèque que l'on retrouve sous une forme beaucoup moins politisée chez ceux qui leur ont succédé.

\section{Le ciblage du public}

Le discours commence systématiquement par le ciblage, l'assignation d'un public qui pourrait bénéficier d'une politique publique locale. Ici, puisque nous étudions le premier chèque culture introduit en France au conseil régional RhôneAlpes à destination des lycéens en 1993-1994, il s'agit donc de cibler la jeunesse, les lycéens, mais certains des acteurs interrogés vont plus loin et trouvent d'eux-mêmes des exemples de public à favoriser. On est ici dans ce que Robert Castel ou Gosta Esping-Andersen définissent comme étant le ciblage ou «targeting » des aides, qui favoriserait l'accès du public ciblé au marché (Castel, 2005, Esping-Andersen, 1990). Cette notion de ciblage revient également systématiquement dans les propos des acteurs non-libéraux interrogés : il est nécessaire qu'une politique publique touche son public. 
Le libéral revendiqué Amaury Nardonne, ancien président de la commission Culture du conseil régional Rhône-Alpes commence l'entretien par une référence au ciblage en précisant que «nous avons voulu donner la chance à cette population [les lycéens], que nous touchons plus spécifiquement au travers de l'action régionale, de pouvoir profiter de ces équipements ${ }^{7}$. »

Hervé Mariton, ancien vice-président en charge de la culture estime que la région « a aussi son public institutionnel qui sont les lycéens, les jeunes de Rhône-Alpes et qu'elle est attentive à leur parcours culturel ${ }^{8}$.»

On retrouve cette notion de ciblage parmi les arguments des successeurs de ces pionniers qui diffuseront le chèque dans d'autres collectivités. Ainsi, un directeur général adjoint d'un département parle des chèques sociaux comme «d'outils ciblant les gens qui étaient dans le besoin ${ }^{9} \gg, \mathrm{le}$ directeur général des services d'un autre département évoque lui aussi que «l'idée était de fédérer quelque chose au niveau des collégiens ${ }^{10}{ }^{\prime}$ pour décrire le genèse du chéquier culture et sport destiné à ce public. Le vice-président socialiste d'un département parle du chèque comme d'un «outil personnalisé, c'est aussi un contact direct de la collectivité avec le territoire, les habitants jeunes ${ }^{11}$.»

Ces collectivités sont beaucoup plus orientées à gauche, conséquence de l'alternance massive de 2004 où toutes les régions furent gérées par des exécutifs socialistes (à l'exception de l'Alsace, seule région qui n'a curieusement jamais eu recours au chèque) sans que cet aspect essentiel du discours ne soit modifié pour autant. La notion de ciblage, l'assignation d'un public à une aide individuelle apparaissent donc comme l'entrée essentielle pour conduire vers la logique du chèque et l'introduction de cet instrument néolibéral.

\section{La promotion du libre choix}

La sémantique glisse ensuite vers ce qui caractérise le mieux le chèque outre-Atlantique dans les nombreux débats sur le sujet, lui donnant son caractère libéral (Greene, 2001, Steuerle, 2000) : la notion de libre choix, de choix individuel qui est exprimée de toutes les façons possibles par les médiateurs néolibéraux interrogés. Ce glissement se retrouve de façon assez systématique, chez les libéraux les plus assumés : la notion de liberté individuelle, censée être fournie par le chèque, est déduite de la définition du «public » ciblé. La liberté est donc forcément «ciblée » pour les élus libéraux interrogés.

La plupart du temps, la notion de libre choix se retrouve beaucoup plus politisée et accompagnée de critique négative (contre ceux qui restreindrait la liberté) chez les libéraux revendiqués que chez les acteurs réputés moins engagés. Ainsi, le Président de la commission Culture du conseil régional Rhône-Alpes indiquait que le libre choix permettait de rendre le pouvoir au citoyen, à l'usager, le transformant en «client » de la politique publique, reprenant ainsi un argument classique du Nouveau Management Public (Hood, 1991, Lacheret, 2015) : " le seul moyen de faire une politique culturelle dans laquelle le politique n'est pas un censeur, c'est de donner le choix au citoyen. C'est de donner le choix au citoyen de subventionner - pas d'acheter 
hein!-de subventionner, l'institution culturelle, le spectacle, le musée $e^{12} . »$

Cette volonté de rendre le pouvoir au citoyen par le biais du libre choix se vérifie également dans les propos d'Hervé Mariton qui l'inscrit dans un clivage politique plus général : "Sur un élément comme le chèque culture, on a un exemple de clivage, qui est assez volontiers un clivage politique : les gens de droite ayant envie d'encourager une pratique individuelle, qui peut être une pratique éclairée, qui peut être une pratique bénéficiant d'un environnement, d'une pédagogie, mais qui à un moment, constate un choix individuel, et au fond, depuis qu'on a mis en place le chèque culture, on a toujours constaté que la gauche avait plutôt envie de ramener le chèque culture vers un usage collectif ${ }^{13}$. »

Charles Millon est le seul parmi les acteurs à relier clairement le chèque à un exemple existant, sans être toutefois factuellement exact : «C'est à partir de ce moment-là que l'idée a germé dans les services et qu'est arrivé le système de chèque culture, qui permettait de respecter le choix des gens. C'est parti en réalité avant, avec le chèque éducation, moi je suis un fana du chèque éducation, qui n'a pas encore été mis en place en France. »

L'ancien président de la région (qui fut brièvement ministre de la Défense) est le premier des acteurs interrogés à se référer explicitement au chèque éducation. Proche d'Alain Madelin, dont il a lu l'ouvrage sur l'éducation paru en 1986, Charles Millon est l'un des acteurs de la «parenthèse libérale » des années 1980 qui a vu la droite française s'intéresser aux idées nouvelles venues notamment d'outre-Manche à travers un foisonnement de clubs, de courants et de think tanks. L'idée de chèque éducation est également présente dans l'ouvrage d'Henri Lepage, Demain le Libéralisme, paru en 1980, qui sera également un carburant intellectuel pour les libéraux français (Lepage, 1980).

Plus loin dans l'entretien, l'ancien président du conseil régional indique que le chèque éducation est d'origine thatchérienne et que l'idée lui a été inspirée par l'exemple britannique, ignorant le lien avec Friedman et les débats très vifs aux États-Unis. Dans l'esprit de Charles Millon, le chèque n'a donc jamais vraiment été testé avant lui et il n'est pas en mesure de citer des références précises (alors que cet outil a été utilisé aux États-Unis dans les années 1960). "Tout jeune étudiant, et même tout jeune thésard, je me souviens quej'étais allé en Grande-Bretagne voir quelles avaient été toutes les procédures qu'avait essayé d'utiliser Margaret Thatcher pour pouvoir mettre en place le chèque éducation, ce qui malheureusement d'ailleurs a échoué là-bas pour une raison simple c'est qu'ils n'ont pas pu licencier les fonctionnaires car à ce moment-là il faut privatiser le tout. Mais autrement c'est à partir de ce moment-là qu'est née cette idée... »

Or, à première vue, cette phrase ne fait pas réellement sens puisque Charles Millon n'a pu être étudiant au moment où Margaret Thatcher était Premier ministre britannique. Toutefois, cela peut correspondre à la période 1970-1974 où elle fut secrétaire d'État à l'Éducation et aux Sciences du Royaume-Uni. Sans réellement s'en apercevoir, Charles Millon établit ici un pont idéal entre Milton Friedman et l'Europe puisqu'il se réfère 
à une période où Friedman conseille les libéraux de nombreux pays du monde (il parviendra notamment à mettre en place un chèque éducation au Chili peu après le coup d'État de Pinochet en 1974.).

Parmi les autres acteurs interrogés, on retrouve également des approximations idéologiques qui montrent toutefois clairement une origine néolibérale du chèque. L'un d'entre eux citant la notion d'ordolibéralisme en donne une définition erronée et confesse ne pas réellement savoir de quoi il s'agit : «Et d'ailleurs le discours actuel de notre président de la République [il parle de Nicolas Sarkozy] m'inquiète, il avait été élu en 2007 sur une rupture de ce point de vue avec l'idéologie dominante, que moi je qualifierais de libérale, mais Millon aimait bien le qualificatif d'ordolibéral', je sais pas ce que ça veut dire moi, parce que de toute façon, il n'y a pas de libéralisme sans ordre... donc et là il y a des discours de Sarko, là... enfin Davos, c'était un discours à la Besancenot, ça n'a aucun sens ${ }^{15}$. »

\section{Des acteurs locaux pour un discours local}

Les acteurs politiques libéraux interrogés ont beau avoir parfois eu des carrières gouvernementales ${ }^{16}$, les propos tenus faisant référence à la théorie libérale ou au néolibéralisme montrent une faiblesse de leur culture académique sur ce point précis. De même, les références et les exemples cités sont très lacunaires. L'hypothèse selon laquelle les clubs de réflexions ou think tanks influenceraient les élus, ou que ces derniers appliqueraient des théories et dupliqueraient des expériences observées à l'étranger n'est pas complètement vérifiée par ces entretiens. Cette influence est certes perceptible mais ne s'inscrit pas dans un discours construit. Les élus ont donc souvent retenu quelques bribes, quelques innovations issues de discours souvent plus complexes et les ont ensuite appliquées localement.

Nous avons ici à faire à des élus locaux, préoccupés par des politiques locales. Ces derniers se sentent libéraux sans réellement pouvoir s'appuyer sur un véritable corpus doctrinaire ni sur une démarche de benchmarking particulier. Même s'il est aisément vérifiable que les élus interrogés ont fréquemment été associés à des courant philosophiques ou à des mouvements de pensée libéraux, ils sont souvent assez peu outillés idéologiquement et gardent une démarche particulièrement pragmatique en utilisant des éléments qu'ils ont parfois pu piocher en assistant à des événements et manifestations organisés au cours de la «parenthèse libérale de 1986 » en France.

De fait, chez les libéraux interrogés, la légitimation du chèque s'opère à travers un discours en trois temps. Les acteurs évoquent d'abord la "nécessité de ciblage d'un public » avant d'y lier la nécessité de «laisser le libre choix» au bénéficiaire qui justifie ensuite l'usage d'un « instrument spécifique ».

Cette structure s'est révélée très efficace une fois débarrassée de son habillage néolibéral revendiqué, et a pu être aisément reprise par d'autres acteurs politiques et administratifs, notamment après une alternance électorale locale. Le discours partant du «public » et de la nécessité de le cibler pour justifier le libre choix puis l'usage du chèque a fini par devenir davantage un discours 
instrumental classique, dépolitisé et neutralisé car ses promoteurs initiaux ont adopté une posture d'élus locaux, revendiquant certes une filiation libérale mais sans être réellement capables de bâtir un argumentaire politique solide sur le sujet.

\section{La structure de l'argumentation, explication de l'acceptation politique du chèque ?}

Les élus libéraux, interrogés de nouveau en 2013, commentant la diffusion du chèque au sein des différentes collectivités locales dont la plupart étaient gérées par des exécutifs de gauche vont, comme c'est assez prévisible, témoigner d'un triomphe du néolibéralisme. Ainsi, un élu libéral, présent lors de la naissance du chèque culture, répond à la question de la diffusion du chèque en soulignant le triomphe des idées libérales par la formule, «la liberté est dévastatrice ${ }^{17}$ », qu'il répétera à de nombreuses reprises au fil de l'entretien et notamment en insistant sur le caractère équitable du chèque : "Alors moi après je considère que c'est un outil libéral puisque c'est un outil qui par définition est non interventionniste, [...] je pense que c'est l'effet dévastateur de la liberté, c'est-à-dire que les systèmes équitables sont toujours préférés aux systèmes égalitaires, qui sont par nature inéquitables. »

De même, Amaury Nardone, en 2010, justifie de nouveau le succès du chèque en utilisant un argumentaire liant confiance et liberté : «Le chèque culture était par nature un outil personnel, pas individualiste, personnel, je me choisis mon livre, je me choisis mon cinoche, et si j'ai envie d'y aller avec mon voisin de droite, j'y vais, mon voisin de gauche, j'y vais, si j'ai envie d'y aller avec les deux, j'y vais, si j'ai envie d'y aller tout seul, j'y vais tout seul, et son succès c'est que c'était un chèque personnel. Ce qui était...le contraire du mépris, c'est le respect, c'est-à-dire que si on vous donne un outil en pensant que vous êtes capable de l'utiliser, déjà, vous vous sentez beaucoup mieux quand même! »

D'autres élus libéraux, interrogés au cours de la même période et commentant le succès du chèque ont une analyse plus mesurée et mettent en avant un point essentiel dans le processus de transition et de neutralisation de l'objet : le fait que le chèque soit un outil de simplification administrative. Ainsi, Charles Millon, revenant sur la diffusion et le succès du chèque en 2013, indique les raisons suivantes : "Cette diffusion s'est faite pour deux raisons, la première c'est que c'est beaucoup plus facile à gérer puisqu'on laisse aux gens le choix, et deuxièmement c'est que la gauche a repris les régions d'une autre manière, c'est-à-dire qu'elle a serré les critères d'attribution. Et en soi, c'est de bonne guerre... $C$ 'est un mode de gestion qui est beaucoup plus strict pour les finances publiques. »

À ses yeux, le chèque n'est donc plus un outil libéral tel qu'il a été conçu au départ puisque son utilisation est davantage encadrée, notamment par l'ajout de critères en restreignant l'usage et l'accès, ce qui en fait un instrument beaucoup plus «neutre » politiquement (Lacheret, 2015).

Ce changement discursif est aisément identifiable, y compris dans la communication institutionnelle de la Région, qui reflète bien les changements dans les discours des acteurs.

Ainsi, en 1998, un dossier de presse présentant le 
chèque culture Rhône-Alpes précise que l'un des objectifs essentiels de cet outil est de : «favoriser l'éducation culturelle et le choix en donnant les moyens aux jeunes de se prendre en charge lorsqu'ils peuvent utiliser le chèque culture de façon autonome ${ }^{18}$. »

Dans ce même document, le chèque est même défini comme une politique culturelle indépendante : "Le chèque culture constitue une véritable pratique culturelle, dont le but est d'amener les jeunes à une bonne appréhension de leur environnement culturel. »

Cette communication institutionnelle reprend les marqueurs libéraux décrits en interne par les acteurs interrogés pendant la période. Le chèque est présenté comme une politique culturelle à part entière et s'éloigne de la notion d'instrument neutre que l'on retrouve chez les acteurs non libéraux.

Quinze ans plus tard, la communication institutionnelle de la région Rhône-Alpes en 2013 à propos du dispositif témoigne d'un changement sémantique évident en soulignant que le dispositif permet un «engagement citoyen, un soutien à l'initiative jeunesse et une aide à l'autonomie ». Dans la presse régionale, l'élue en charge de la jeunesse définit la carte $\mathrm{MRA}^{19}$, successeur du chèque culture comme «l'outil le plus connu et reconnu par les jeunes et les familles ${ }^{20}$. " Cette évolution dans le choix des termes a permis de donner une dimension technique et utilitariste au chèque, faisant par ce biais disparaître son origine néolibérale et donc a rendu beaucoup plus simple sa diffusion en en faisant un outil plutôt qu'une politique à part entière. Les médiateurs néolibéraux à l'origine de son introduction interrogés en conviennent tous. L'adoucissement considérable du discours libéral apparaît évident chez les acteurs du chèque appartenant à des majorités de gauche.

\section{Un glissement sémantique qui ne change rien à la structure discursive}

Ainsi, on retrouve des termes mettant beaucoup moins en avant le libre choix et mettant l'accent sur le ciblage du public, dans les propos de la conseillère régionale déléguée à la Jeunesse de la majorité de gauche en Rhône-Alpes en 2013 qui évoque : « un outil qui était au début très "lycées » avec les manuels scolaires, ensuite on est passé au développement personnel, avec l'accès a la culture, l'accès aux loisirs, l'accès aux sports [...] c'est vraiment en train de passer sur une carte qui ne serait plus la carte du lycéen, mais celle de tous les jeunes Rhône-Alpes ${ }^{21}$. »

Il en est de même au sein d'autres acteurs comme ce DGS d'un département : "on cherchait pour les collégiens quelque chose de rassembleur ${ }^{22}$ ", ou ce cadre du service Jeunesse de la région Rhône-Alpes qui parle d'un « outil qui matérialise les interventions de la région auprès $d u$ public des jeunes dont la tranche d'âge est de 15 à 25 ans $^{23}$. »

Derrière le côté utilitariste, on retrouve un champ lexical davantage orienté vers l'émancipation, l'autonomie, qui est identifiée chez tous les élus interrogés (tous appartiennent à des majorités de gauche) après 2010 et qui n'ont pas particulièrement réfléchi à l'origine et au sens politique du chèque. Au fond, ce vocabulaire n'est qu'une 
évolution des propos des médiateurs néolibéraux à l'origine du chèque, la promotion du «libre choix» se transformant en « autonomie », le côté «libéral » étant plus volontiers défini comme " émancipateur " même si, en définitive, les caractéristiques de l'objet n'ont pas changé.

Cette transformation est même accentuée et reconnue par certains acteurs administratifs qui identifient un «tournant » dans la façon d'appréhender le chèque. Ainsi, le chef du service gérant la carte MRA au conseil régional Rhône-Alpes en 2012, qui était attaché de presse de l'exécutif précédent, évoque ce changement de perception de l'outil en intégrant ce terme qui remplace la valorisation du choix et ouvre l'ère de l'" autonomie » : «Maintenant, comme je vous le disais, j'ai l'impression qu'on est peut-être à une fin de cycle, on a ajouté de nouveaux publics, de nouvelles options donc on est peut-être à l'aube de quelque chose de nouveau avec cette réflexion sur l'autonomie ${ }^{24}$. »

Un autre cadre administratif régional répond à une interrogation sur le caractère libéral du chèque en mobilisant immédiatement le vocabulaire alternatif utilisé dans la communication institutionnelle de la région : "Par rapport au reproche que la carte soit un outil libéral, c'est vrai, mais c'est un acte d'autonomisation du jeune, vraiment pour le laisser libre de choisir ${ }^{25}$.»

\section{$* * *$}

L'objet très politisé dans les discours à la fin des années 1990 des deux côtés de l'Atlantique (Greene 2008, Carnoy, 2001), volontiers présenté comme libéral par ses promoteurs au sein des col- lectivités locales est désormais présenté sous un angle beaucoup plus neutre, reprenant toutefois plusieurs éléments importants caractéristiques du néolibéralisme.

Tout d'abord, le chèque reste un outil de ciblage du public quel que soit l'acteur interrogé. Cette manière de définir le public est encore plus visible avec les chèques sociaux, destinés à un public rencontrant des difficultés et lui permettant de bénéficier d'aides très encadrées. Ainsi, à de nombreuses reprises, les élus en charge de la solidarité et les agents des collectivités définissent en premier lieu le chèque comme un outil d'assignation d'un public.

Ensuite, le chèque permet l'«autonomie » et l'«émancipation», nouveaux termes plus neutres pour évoquer le «libre choix».

Bien entendu, les termes employés pour définir l'utilité du chèque ne sont pas ceux de Michel Foucault mais nous sommes clairement ici dans une logique d'accès à un service encadré au sein de ce que l'on peut appeler un quasi-marché (Glennester, Le Grand, 1995). En d'autres termes, pour revenir au bénéficiaire du chèque social, celui-ci n'a pas les moyens d'accéder au marché des fournisseurs d'aides sociales et doit donc être aidé par la puissance publique qui ne lui fournit donc pas l'aide directement, mais lui donne le moyen d'accéder à cette aide.

Ce changement sémantique entre les médiateurs néolibéraux et les acteurs contemporains ne doit toutefois pas masquer le fait que le caractère néolibéral du chèque est on ne peut plus présent, le projet de pass culture du gouvernement français 
s'inscrit d'ailleurs parfaitement dans une logique d'aide à l'accès au marché. Il est d'ailleurs notable que lors des différentes prises de paroles de la ministre de la Culture sur le sujet, le discours néolibéral se retrouve aisément. Ainsi, dans le Monde du 6 mars 2018, Francoise Nyssens indique que l'objectif du pass culture est de : « laisser le jeune autonome dans ses choix », ajoutant plus loin que «traditionnellement, le ministère passe par ses institutions pour s'adresser aux citoyens, comme par exemple nos musées avec des politiques de gratuité. Avec le pass culture, c'est l'inverse : on donne à l'usager les moyens d'être " autoprescripteur », de se diriger vers l'offre de son choix, publique ou privée, sans distinction ${ }^{26}$. ”

Nous sommes ici dans la logique du chèque éducation où la puissance publique ouvre à un public ciblé la possibilité d'un choix encadré (on peut mentionner notamment les " charter schools » accréditées par l'État) (Molnar, 1998, 2018).

Les médiateurs néolibéraux du chèque au sein des collectivités territoriales françaises ont donc su développer un discours très politisé et militant au départ en apparence dont la structure a ensuite pu être reprise par leurs successeurs qui ont diffusé l'instrument «chèque » en le développant techniquement pour l'adapter aux évolutions technologiques (transformation en carte à puce dans de nombreuses régions, en application mobile dans le projet gouvernemental de pass culture) sans pour autant en modifier la structure.

Cette approche très localiste des pionniers comme Charles Millon et sa majorité régionale en 19931994 a permis d'implanter durablement le chèque dans les politiques de versement d'aides ciblées à un public assigné en France. Elle a aussi permis d'éviter l'écueil américain. En effet, outreAtlantique, le « voucher » est devenu un marqueur politique fort et une forme d'épouvantail qui est régulièrement brandi par les démocrates, notamment à travers l'image du chèque éducation.

Ainsi, les néolibéraux français ont su tirer les leçons des difficultés d'implantation du chèque en inscrivant ce dernier dans un discours utilitariste « acceptable » qui a ensuite pu être digéré par leurs successeurs qui n'ont eu qu'à le modifier à la marge et notamment en «neutraliser » le champ sémantique pour rendre un outil néolibéral par excellence parfaitement accepté dans le paysage des politiques publiques françaises. 


\section{$R \cdot E ́ F \cdot E \cdot R \cdot E \cdot N \cdot C \cdot E \cdot S$}

Marta BALTODANO, "Neoliberalism and the demise of public education : the corporatization of schools of education", International Journal of Qualitative Studies in Education, 25:4, 2012, p. 487-507.

Kevin BROOKES, Diffusion et transformation du néo-libéralisme en France des années 1960 aux années 1980, L'Association pour la Liberté et le Progrès Social et les "Nouveaux Économistes », Institut d'Études politiques de Grenoble, Grenoble, 2012.

Martin CARNOY (dir.), School vouchers, examining the evidence, Economic policy institute, 2008.

Robert CASTEL, « Devenir de l'État providence et travail social $»$ in Jacques Ion (dir), Le travail social en débat [s], La Découverte, 2005, p. 27-49.

Frédéric COLIN, «Les « bons d'échange » ou « vouchers » de service public », Revue Internationale des Sciences Administratives, $\mathrm{n}^{\circ} 71$, 2005, p. 19-34.

Patricia COMMUN, Les Ordolibéraux : Histoire d'un libéralisme à l'allemande, Paris, Les Belles Lettres, 2016.

David DOLOWITZ, David MARSH, "Learning from abroad : the role of policy transfer in contemporary policy making", Governance, $\mathrm{n}^{\circ} 13$, 2000, p. 5-24.

Anna DURNOVA, Philippe ZITTOUN, « Les approches discursives des politiques publiques. Introduction », Revue française de science politique, 2013/3 (Vol.63), p. 569-577.

Gosta ESPING-ANDERSEN, The Three Worlds of Welfare Capitalism, Princeton University
Press, 1990.

Michel FOUCAULT, Naissance de la biopolitique, Paris, Gallimard, 2004.

Milton FRIEDMAN, Capitalism and freedom, University of Chicago Press, 1962.

Milton FRIEDMAN, "The Role of Government in Education", in Robert Solo (dir), Economics and the Public Interest, Rutgers University Press, 1955, p. 127.

Howard GLENNERSTER, Julian Le GRAND, "Le développement des quasi-marchés dans la protection sociale », Revue Française d'Economie, vol 10, 1995, p. 111-135.

Jay GREENE, Paul PETERSON et Jiangtao DU, "School choice in Milwauke : A randomized experiment", in Jay Greene, An evaluation of the Florida A-Plus accountability and school choice program. New York, Center for Civic Innovation, Manhattan Institute, 2001.

Jay GREENE, Why America Needs School Choice?, Encounter Broadside, 2011.

Christopher HOOD, "A public management for all seasons ?", Public Administration, Vol 69, 1991, p. 3-19.

Sabine LACERENZA, «L'impensé des études sur les effets des politiques de tarification L'exemple du chèque culture en région RhôneAlpes », in Olivier Donnat, Sylvie Octobre (dir), Les publics des équipements culturels-Méthodes et résultats d'enquêtes, Paris, Ministère de la Culture et de la Communication, Département des Études et de la Prospective, collection Les Travaux du DEP, 2001.

Arnaud LACHERET, « Le chèque (voucher), instrument néolibéral et/ou innovation institutionnelle ? », Entreprendre \& Innover, $\mathrm{n}^{\circ}$ 32, 2017. Arnaud LACHERET, « Le voucher : instrument de la libéralisation de l'aide sociale en France? », 
Politiques et management publics, 2015. Arnaud LACHERET, L'aide sociale par le chèque : genèse et mise en æuvre des politiques sociales et culturelles " ciblées 》 des collectivités territoriales, Science politique, Université de Grenoble, 2014.

Arnaud LACHERET, " L'évaluation comme instrument d'effacement du sens politique : la controverse autour de l'évaluation des schools vouchers américains ", Revue française d'administration publique, ENA, 2013.

Arnaud LACHERET, « Le chèque comme outil du Nouveau Management Public dans les collectivités locales françaises ", Gestion et Management public, AIRMAP, nº, 2015, p.81-100.

Pierre LASCOUMES, Patrick Le Galès (dir), Gouverner par les instruments, Presses de Science po, 2004.

Patrick MAC EWAN, "The Potential Impact of Vouchers", Peabody Journal of Education, ${ }^{\circ} 79$, 2004, p. 57-80.

Alain MADELIN, Pour libérer l'école : l'enseignement à la carte, Robert Laffont, 1984.

John Stuart MILL, De la Liberté, Guillaumin, 1990 (édition originale 1860).

Alex MOLNAR, Giving kids the Business, the commercialization of America's schools, Taylor and Francis, 2018.

Jean-Paul NAVAILLES, La famille ouvrière dans l'Angleterre Victorienne, Champ Vallon, 1983.

Alan PEACOCK, "Public Patronage and Music: an Economists View", Three Banks Review, mars 1968, p. 1-19.

Alan PEACOCK, "Welfare economics and public subsidies to the arts", Manchester School of Economic and Social Studies, n³7, 1969, p. 323-335. Alan PEACOCK, "Public financing of the Arts in England", Fiscal Studies, n²1, 2000, p. 171-205.
Alan PEACOCK, Ilde RIZZO, Cultural Economics and Cultural Policies, Springer, 1994.

Jérôme PERRIER, « La parenthèse libérale de la droite française des années 1980. Le phénomène politique de la «bande à Léo» ou l'échec de la promotion d'un libéralisme contre l'État ", Histoire@Politique, n²5, 2015.

Paul PETERSON, Bryan HASSEL (dir), Learning from school choice, Brookings Institute, 1998, p. 335-356.

François ROUET (dir), "Cartes et chèques culture : dispositifs et mise en œuvre », Culture études, $\mathrm{n}^{\circ} 6,2009$, p. 1-8.

Guy SAEZ, Arnaud LACHERET, « La politique culturelle régionale sur la voie de l'autonomie » in Bernard Jouve, Vincent Spenlehauer, Philippe Warin, La Région Laboratoire politique, une radiographie de Rhône-Alpes, La Découverte, 2001.

Lester SALAMON, "The New Governance and the Tools of Public Action: An introduction", Fordham Urban Law Journal, vol 28, 2000, p. 1611-1674.

Eugene STEUERLE, Éric TWOMBLY, "Vouchers", in Lester, Salamon (dir), The Tools of Government: A Guide to the New Governance, Oxford University Press, 2002, p. 445-465.

Eugene STEUERLE (dir), Vouchers and the provision of public services, Brookings Institute, 2000.

Gary STURGESS, Ivana BODROZA, Redeeming vouchers in Public Services, Serco Institute, 2011.

Danny WEIL, School vouchers and privatization: a reference handbook, ABC-CLIO, 2002. 


\begin{abstract}
$\mathrm{N} \cdot \mathrm{O} \cdot \mathrm{T} \cdot \mathrm{E} \cdot \mathrm{S}$
1. https://www.lemonde.fr/politique/article/2018/03/06/avec-le-passe-francoise-nyssen-doit-definir-ce-qu-est-la-culture-pour-lesjeunes_5266271_823448.html
\end{abstract}

2. Entretien avec un membre de l'exécutif, $1^{\text {er }}$ mars 1999.

3. Entretiens avec Charles Millon, 15 avril 2000 et 8 mars 2010 .

4. Parmi d'innombrables articles, on peut relever celui de Time, "Betsy DeVos Wants to Spend Millions on School Vouchers. Two New Studies Say They Don't Actually Work", Time, 27 juin 2017.

5. «Les instruments de l'action publique ne sont pas des outils axiologiquement neutres, et indifféremment disponibles. Ils sont au contraire porteurs de valeurs, nourris d'une interprétation du social et de conceptions précises du mode de régulation envisagé. Un instrument d'action publique constitue un dispositif à la fois technique et social qui organise des rapports sociaux spécifiques entre la puissance publique et ses destinataires en fonction des représentations et des significations dont il est porteur », Pierre Lascoumes, Patrick Le Galès, «L'action publique saisie par les instruments ", in Pierre Lascoumes, Patrick Le Galès (dir), Gouverner par les instruments, Presses de la Fondation nationale des sciences politiques, 2004, p. 13.

6. Les acteurs qualifiés de contemporains sont ceux, souvent de gauche, qui ont succédé aux libéraux revendiqués.

7. Entretien réalisé le 1er mars 1999.

8. Entretien réalisé le 15 avril 2000.

9. Entretien réalisé le 31 janvier 2013.

10. Entretien réalisé le 11 juillet 2011.

11. Entretien réalisé le 21 février 2012.
12. Op. cit.

13. Op. cit.

14. L'ordolibéralisme est une doctrine née en Allemagne au début du $\mathrm{XX}^{\mathrm{e}}$ siècle qui part du principe que l'ordre économique repose sur un choix juridique fondamental que les ordolibéraux analysent à travers la notion de «constitution économique » (Commun, 2016).

15. Entretien avec un membre de la majorité régionale Rhône-Alpes, 28 avril 1999.

16. Outre Charles Millon qui fut ministre de la Défense entre 1995 et 1997, Hervé Mariton fut très brièvement ministre de l'Outre Mer en 2007.

17. Entretien avec un conseiller régional Rhône-Alpes effectué le $1^{\text {er }}$ février 2013.

18. Conseil régional Rhône-Alpes, « Le chèque culture, une initiative unique en France », Dossier de presse, Direction de la communication, janvier 1998

19. Dénomination contractant le verbe « aimer», puis les initiales de la Région Rhône-Alpes.

20. http://www.drome-hebdo.fr/blog/2012/06/04/ $1 \mathrm{ance}$ e n t - d e - $1 \mathrm{a}-\mathrm{n}$ o u ve $11 \mathrm{e}-\mathrm{c}$ arte m\%E2\%80\%99ra-2012-2013/

21. Entretien réalisé le 27 septembre 2012.

22. Entretien réalisé le 11 juillet 2011.

23. Entretien réalisé le 20 avril 2013.

24. Entretien réalisé le 8 octobre 2012.

25. Entretien réalisé le 15 juin 2012.

26. https://www.lemonde.fr/politique/article/2018/03/06/avec-le-passe-francoise-nyssen-doit-definir-ce-qu-est-la-culture-pour-lesjeunes_5266271_823448.html 


\title{
$R \cdot E ́ \cdot S \cdot U \cdot M \cdot E ́$
}

Alors que le gouvernement français s'apprête à lancer un pass culture destiné aux jeunes de 18 ans, il est proposé de revenir sur les multiples dispositifs similaires lancés depuis les années 1990 par les collectivités locales françaises en examinant leur filiation néolibérale. Nous montrerons dans cet article que si les élus locaux pionniers du chèque affichaient sans complexe leurs visées néolibérales, leurs successeurs ont eu une approche bien plus mesurée et utilitariste permettant d'éviter tout débat politique de fond sur cet instrument. Les médiateurs néolibéraux du chèque au sein des collectivités territoriales françaises ont ainsi su développer un discours très politisé et militant au départ dont la structure a ensuite pu être reprise par leurs successeurs qui ont diffusé l'instrument « chèque » en le développant de façon purement formelle sans pour autant en modifier la structure.

\begin{abstract}
Whilst The French Government is about to launch a cultural voucher targeting the 18 -years-olds, we aim to have a glance at the many similar tools developed during the 1990s by the French local authorities focusing on their neoliberal origins. We will show in the following paper that if the local officials acting as trailblazers of the use of vouchers were clearly neoliberal, their successors endeavoured to define them as a functional tools, avoiding some hard political debates on its depth nature. The neoliberal mediators of the vouchers within local authorities used first to develop a very politicized discourse whose structure has been kept by their successors who spread vouchers throughout the French regions, developing it only formally without modifying its political meaning.
\end{abstract}

